\title{
Hydrogen-terminated defects in ion-implanted silicon probed by monoenergetic positron beams
}

\author{
Akira Uedono, Toshiki Mori, Kunitomo Morisawa, and Kouichi Murakami \\ Institute of Applied Physics, University of Tsukuba, Tsukuba, Ibaraki 305-8573, Japan \\ Toshiyuki Ohdaira, Ryoichi Suzuki, and Tomohisa Mikado \\ National Institute of Advanced Industrial Science and Technology, Tsukuba, Ibaraki 305-8568, Japan \\ Kunie Ishioka and Masahiro Kitajima \\ Material Engineering Laboratory, National Institute for Materials Science, Tsukuba, \\ Ibaraki 305-0047, Japan \\ Shunichi Hishita, Hajime Haneda, and Isao Sakaguchi \\ Advanced Materials Laboratory, National Institute for Materials Science, Tsukuba, Ibaraki 305-0044, Japan
}

(Received 18 July 2002; accepted 6 December 2002)

\begin{abstract}
Hydrogen-terminated vacancies in $\mathrm{Si}^{+}$-implanted $\mathrm{Si}$ were studied by means of positron annihilation. After the $\mathrm{Si}^{+}$-ion implantation, hydrogen atoms were introduced into the damaged region using a hydrogen plasma [hydrogen-atom treatment (HAT)]. Monoenergetic positron beams were used to measure Doppler broadening spectra of the annihilation radiation and the lifetime spectra of positrons. It was found that the line shape parameter, $S$, corresponding to the annihilation of positrons trapped by vacancy-type defects, decreased after HAT. This was attributed to the trapping of positrons by H-decorated vacancy-type defects. Isochronal annealing experiments revealed a strong correlation between positron annihilation parameters and Raman intensities of $\mathrm{Si}-\mathrm{H}$, suggesting that hydrogen atoms are released from vacancy-type defects after annealing at $600{ }^{\circ} \mathrm{C}$. (C) 2003 American Institute of Physics. [DOI: 10.1063/1.1542923]
\end{abstract}

\section{INTRODUCTION}

It is important to study the behavior of hydrogen in $\mathrm{Si}$ because it affects the electrical properties of $\mathrm{Si}$ devices. ${ }^{1}$ During the processing steps of semiconductor devices, $\mathrm{Si}$ wafers interact with a large amount of hydrogen. For example, bond-and-etch-back processes used in the fabrication of silicon-on-insulator wafers involve implantation of high doses of hydrogen or annealing in hydrogen atmosphere. ${ }^{2,3}$ Since silanes are the major source for epitaxial growth of $\mathrm{Si}$ films, the surface of the film is covered by hydrogen ${ }^{4}$ Raman scattering and infrared absorption are known to be useful tools for probing hydrogen in $\mathrm{Si}$; several scattering or absorption peaks were identified as $\mathrm{H}_{2}$ molecules trapped at tetrahedral $\left(T_{d}\right)$ sites or trapped by platelets, voids, point defects, etc. ${ }^{1,5-9}$ Hydrogen terminates dangling bonds of vacancy-type defects, ${ }^{8,9}$ and also interacts with interstitials. ${ }^{10}$ Although intensive studies have been carried out, the interaction between hydrogen and process-induced defects is still unresolved because of the lack of techniques that are sensitive to the presence of point defects in the subsurface region. Positron annihilation is one established technique for investigating defects in semiconductors. ${ }^{11}$ A slow/monoenergetic positron beam can be used to adjust the implantation profile of positrons in the region of interest in a sample by accelerating the positrons to a certain energy. In general, the incident energy of positrons varies from several electron volts to $30-50 \mathrm{keV}$, and the regions that can be sampled by such positrons can vary from the sample surface to depths of the order of microns. Defects introduced by hydrogen plasma or $\mathrm{H}^{+}$implantation have been investigated by this technique, ${ }^{12-17}$ which showed that positrons are a useful probe for studying hydrogen-related defects in Si. The present study used a monoenergetic positron beam to investigate the $\mathrm{H}$-terminated vacancies in $\mathrm{Si}^{+}$-implanted $\mathrm{Si}$.

When a positron is implanted into condensed matter, it annihilates with the electron and, emits two $511 \mathrm{keV} \gamma$ rays. ${ }^{11}$ Those annihilation $\gamma$ rays are broadened by the momentum component of the annihilating electron-positron pair $p_{L}$, which is parallel to the direction of the $\gamma$ rays. The energy of the $\gamma$ rays is given by the relation $E_{\gamma}=511$ $\pm \Delta E_{\gamma} \mathrm{keV}$. The Doppler shift, $\Delta E_{\gamma}$, is given by the relation $\Delta E_{\gamma}=p_{L} c / 2$, where $c$ is the speed of light. A freely diffusing positron may be localized in a vacancy-type defect because of Coulomb repulsion from ion cores. Because the momentum distribution of electrons in such defects differs from that in bulk materials, these defects can be detected by measuring Doppler broadening spectra of annihilation radiation. The resultant change in the Doppler broadening spectra is characterized by the $S$ parameter, which mainly reflects the change due to the annihilation of positron-electron pairs with a low momentum distribution. The lifetime of positrons trapped by vacancy-type defects increases because of the reduced electron density in such defects. Information obtained by measuring the lifetime spectra of positrons is also useful for identifying vacancy-type defects. ${ }^{11}$

\section{EXPERIMENT}

The samples used in the present experiments were undoped $\mathrm{Si}(100)$ wafers (resistivity: $>50 \Omega \mathrm{cm}$ ) grown by the floating-zone (FZ) method. $\mathrm{Si}^{+}$ions were implanted at 200 
$\mathrm{keV}$ up to a dose of $2 \times 10^{14} \mathrm{~cm}^{-2}$ at room temperature. This dosage is lower than the critical dose sufficient for creation of an amorphous region. The implanted samples were treated with atomic hydrogen at $190{ }^{\circ} \mathrm{C}$ for $30 \mathrm{~min}$ in a downstream region of hydrogen plasma. During this treatment, the samples were placed $60 \mathrm{~cm}$ from the plasma so that the activated species in the plasma do not damage the sample surface. This hydrogen-atom treatment (HAT) was also performed on the $\mathrm{Si}^{+}$-implanted sample after being annealed at $435{ }^{\circ} \mathrm{C}$. Raman scattering spectra of the samples were measured at room temperature. Details of the HAT and Raman measurements are described elsewhere. ${ }^{7,8}$ After HAT, the samples were isothermally annealed in a $\mathrm{N}_{2}$ atmosphere at $190-800{ }^{\circ} \mathrm{C}$ for $30 \mathrm{~min}$.

A monoenergetic positron beam was used to measure the Doppler broadening spectra of annihilation radiation as a function of incident positron energy $E$. For each value of $E$, a spectrum with approximately $1 \times 10^{6}$ total counts was measured and characterized by the $S$ parameter; the center region of the spectra was defined as $511 \pm 0.75 \mathrm{keV}$. The relationship between $S$ and $E$ was analyzed by VEPFIT, a computer program developed by van Veen et al. ${ }^{18}$ Details of the application of VEPFIT for ion-implanted $\mathrm{Si}$ are described elsewhere. ${ }^{19}$ In the fitting procedure, the region sampled by positrons was divided into several blocks. VEPFIT was used to determine the fraction of positrons annihilated in each block and the corresponding $S$ parameter. The $S-E$ curve was fit into the following equation:

$$
S(E)=S_{s} F_{s}(E)+\sum_{i=1}^{n} S_{i} F_{i}(E),
$$

where $F_{s}(E)$ is the fraction of positrons annihilated at the surface and $F_{i}(E)$ is that in the $i$ th block $\left[F_{s}(E)+\sum F_{i}(E)\right.$ $=1]$. $S_{S}$ and $S_{i}$ are the $S$ parameters corresponding to, respectively, annihilation of positrons on the surface and that in the $i$ th block. To determine the annihilation characteristic of positrons trapped by H-terminated vacancies, we used a coincidence-detection system ${ }^{11}$ to obtain Doppler broadening spectra. The spectra were measured by a two-dimensional multichannel analyzer, and the diagonal cross section of the spectra was obtained with an energy width of $1.3 \mathrm{keV}$.

A pulsed monoenergetic positron beam was used to obtain the lifetime spectra of positrons. ${ }^{20}$ Approximately 1 $\times 10^{6}$ counts were accumulated for each lifetime spectrum. The lifetime spectrum of positrons, $S_{\mathrm{LT}}(t)$, is given by

$$
S_{\mathrm{LT}}(t)=\sum \lambda_{i} I_{i} \exp \left(-\lambda_{i} t\right)
$$

where $\lambda_{i}$ and $I_{i}$ are, respectively, the annihilation rate of positrons of the $i$ th component and their intensity. The lifetime of positrons, $\tau_{i}$, is given by $1 / \lambda_{i}$. The lifetime spectra were analyzed with a time resolution (full width at half maximum) of approximately 250 ps by a computer program called RESOLUTION. ${ }^{21}$

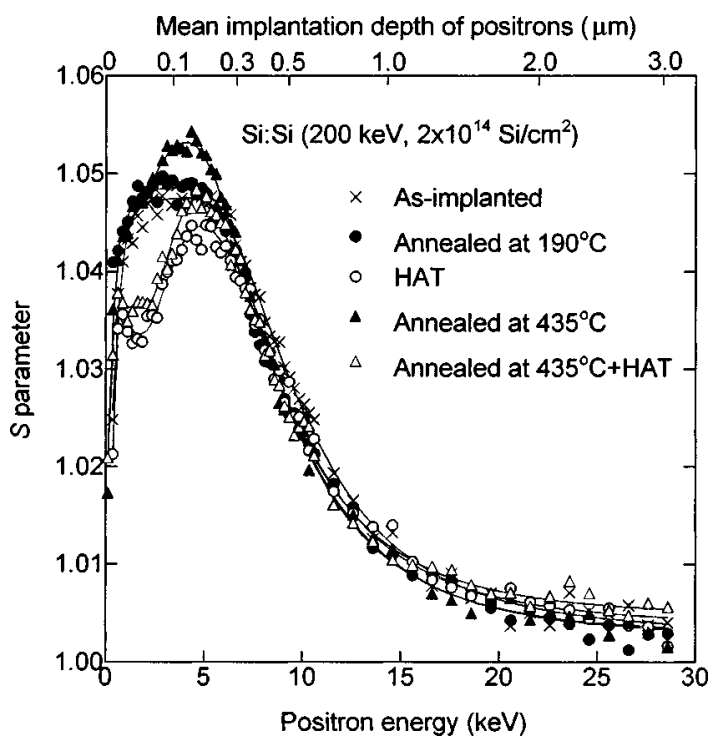

FIG. 1. $S$ parameter as a function of incident positron energy $E$ for $\mathrm{Si}^{+}$- implanted $\mathrm{Si}$ before and after hydrogen atom treatment. Results for the samples annealed at $435{ }^{\circ} \mathrm{C}$ before HAT are also shown. For the samples after HAT, the valley observed in the $S-E$ curve $(E \cong 2 \mathrm{keV})$ is associated with the annihilation of positrons trapped by H-terminated vacancies. The solid curves are fits of Eq. (1) to the experimental data.

\section{RESULTS AND DISCUSSION}

\section{A. Annihilation characteristic of positrons trapped by} hydrogen-terminated vacancies

The $S$ parameters of $\mathrm{Si}^{+}$-implanted $\mathrm{Si}$ before and after HAT are shown in Fig. 1 as a function of $E$. The value of $S$ was normalized to that for bulk Si. The corresponding mean implantation depth of positrons is shown on the upper horizontal axis. Figure 1 shows that $S$ approaches a constant value at high $E(>25 \mathrm{keV})$. This indicates that, in this energy range, almost all positrons annihilate in the substrate without diffusing back to the subsurface region. The observed increase in $S$ at $E \cong 4 \mathrm{keV}$ is due to the trapping of positrons by vacancy-type defects introduced by $\mathrm{Si}^{+}$implantation. ${ }^{19}$ For the as-implanted sample, the lifetime spectrum of positrons was measured at $E=2 \mathrm{keV}$, at which almost all positrons annihilate from the trapped state by defects. The spectrum was analyzed under the assumption of one annihilation mode, and the lifetime of positrons was obtained as 302 \pm 3 ps. Since this lifetime is close to the calculated lifetime of positrons trapped by divacancies, ${ }^{22,23}$ the mean size of the open volume of vacancy-type defects is close to that of divacancies. In the case of the sample that underwent HAT (hereafter referred to as the HAT sample), the value of $S$ at $E \cong 2 \mathrm{keV}$ was found to be smaller than that of the asimplanted sample. The observed decrease in $S$, therefore, can be attributed to the interaction between vacancy-type defects and hydrogen. The decrease in $S$ due to HAT was also observed in the case of the sample annealed at $435{ }^{\circ} \mathrm{C}$, which is higher than the annealing temperature of divacancies in $\mathrm{Si}$ $\left(200-250{ }^{\circ} \mathrm{C}\right){ }^{24}$ Since ion implantation introduces a variety of defects, no large change in the value of $S$ before or after annealing out the divacancies was observed. ${ }^{19,25}$ 


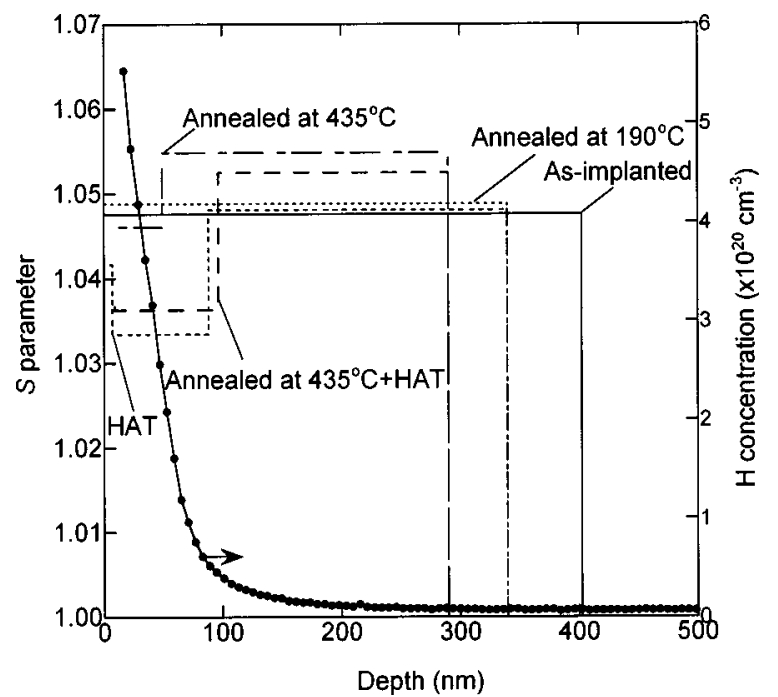

FIG. 2. Depth profiles of $S$ for $\mathrm{Si}^{+}$-implanted $\mathrm{Si}$ before and after HAT obtained from analysis of the $S-E$ curves in Fig. 1. A depth profile of hydrogen measured by SIMS is also shown.

The $S-E$ curves shown in Fig. 1 were fit using VEPFIT. In the fitting procedure, the region sampled by the positrons was divided into two, three, or four blocks. The diffusion length of positrons, $L_{d}$, in the block located in the deepest region was fixed to $L_{d}$ for defect-free $\mathrm{Si}(200 \mathrm{~nm}){ }^{19,26}$ This means that the trapping of positrons by defects in this block is negligible. The solid curves shown in Fig. 1 are the results of fit. The value of $L_{d}$ in the first block, $L_{d 1}$, was derived as about $5 \mathrm{~nm}$. This short diffusion length of positrons suggests that almost all positrons implanted into the first block annihilate from the trapped state. ${ }^{19}$ Thus, $S_{1}$ is considered to be the characteristic value of $S$ for the annihilation of positrons trapped by defects in the first block. In the fitting procedure, it was assumed that $L_{d}$ in the blocks below the first one (except the deepest block) is the same as $L_{d 1}$. Since the $S$ $-E$ curves are represented well by this model, the derived value of $S_{i}$ is likely to be related to the annihilation of positrons trapped by defects in the $i$ th block.

The depth distributions of $S$ obtained are shown in Fig. 2. Using secondary ion mass spectroscopy (SIMS), we measured the depth distribution of hydrogen in the as-implanted HAT sample, and the result is also shown in Fig. 2. After 190 ${ }^{\circ} \mathrm{C}$ annealing, the damaged region shifts toward the surface, but $S_{1}$ does not significantly change. The value of $S$ increases after annealing at $435{ }^{\circ} \mathrm{C}$, suggesting clustering of vacancytype defects. For the as-implanted sample after HAT, the region with a small value of $S(10-100 \mathrm{~nm})$ matches the depth distribution of hydrogen. This suggests that HAT decreases the characteristic value of $S$ in the damaged region. The same is true for the sample annealed at $435^{\circ} \mathrm{C}$.

To interpret the change in Doppler broadening spectra by HAT, the ratio of the coincidence spectra was calculated. Figure 3 plots the Doppler broadening spectrum that corresponds to positron annihilation from the free state on the left-hand axis; the horizontal axis shows $p_{L}\left(m_{0}\right.$ is the rest of the mass of an electron). It can be seen that the spectrum mainly consists of a narrow and a broad component. The

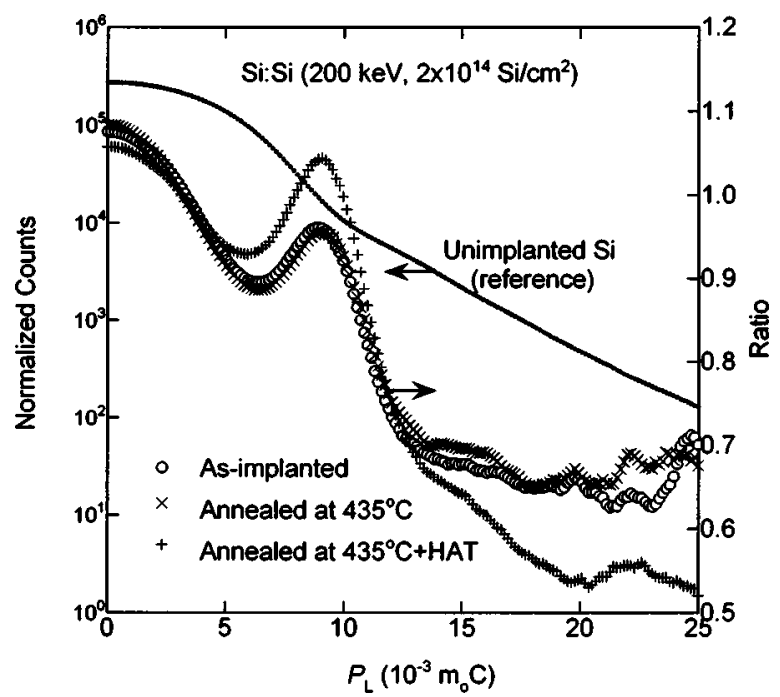

FIG. 3. Doppler broadening spectrum of positrons annihilated from the free state in $\mathrm{Si}$ (used as a reference). Ratio curves of Doppler broadening spectra for the samples before and after annealing at $435^{\circ} \mathrm{C}$ and the HAT sample are also shown; each curve corresponds to the annihilation of positrons trapped by vacancy-type defects or H-terminated defects.

broad component observed at $p_{\mathrm{L}}>10 \times 10^{-3} m_{0} c$ corresponds to the annihilation of positrons with core electrons. This spectrum can be used as a reference. The ratio of the Doppler broadening spectrum measured at $E=2 \mathrm{keV}$ for the sample before and after annealing at $435^{\circ} \mathrm{C}$ and the sample after HAT to the reference spectrum is plotted on the righthand axis in Fig. 3. The overall behavior of the ratio curves for the sample before annealing is similar to that reported by Tang and co-workers. ${ }^{27,28}$ According to Tang and co-workers, ${ }^{27,28}$ the origin of the pronounced peak around $9 \times 10^{-3} m_{0} c$ is slowly varying momentum density of dangling-bond electrons over rapidly decreasing momentum density around the Jones zone boundary of $\mathrm{Si}$.

In Fig. 3, the intensity of the peak around 9 $\times 10^{-3} m_{0} c$ increased after HAT. Using a full-potential and all-electron first-principles method, Tang et al. ${ }^{27}$ calculated Doppler broadening profiles for $\mathrm{Si}$ containing oxygenvacancy complexes. They found that the intensity of the peak around $9 \times 10^{-3} m_{0} c$ becomes significant with an increase in the number of oxygen atoms coupled with divacancies. This was attributed to the fact that the dangling-bond wave functions dominated by oxygen atoms are more localized than those dominated by $\mathrm{Si}$ atoms. ${ }^{27,28}$ In the present experiment, therefore, the observed increase in peak intensity can also be attributed to the annihilation of positrons with electrons localized in vacancy-type defects, and such electrons are introduced by coupling of the defects and hydrogen atoms. This causes a decrease in the annihilation probability of positronelectron pairs with a low-momentum distribution $\left(p_{L}=0\right)$, and results in a decrease in the $S$ value. Raman studies have revealed that HAT produces several Raman lines around $2000 \mathrm{~cm}^{-1}$, which were identified as H-terminated open volumes. ${ }^{1,8,9}$ Those results also support the above discussion.

After HAT, the ratio in the high momentum region $\left(>15 m_{0} c\right)$ is smaller than that before HAT. The effect of 
HAT on the ratio curves of the as-implanted sample without pre-annealing was identical to that on the pre-annealed sample. According to the calculation, ${ }^{27}$ major electrons annihilate with positrons in this region are $2 p$ and $2 s$ core electrons of $\mathrm{Si}$. In H-decorated vacancies, a positron is likely to be localized at the center of the open volume because of the repulsion between a positron and hydrogen atoms, and this could be the origin of the observed decrease in the annihilation probability of positrons with core electrons of $\mathrm{Si}$ atoms. For the sample annealed at $435{ }^{\circ} \mathrm{C}$, after HAT, the lifetime of positrons at $E=2 \mathrm{keV}$ was $324 \pm 1$ ps. Because the lifetime of positrons trapped by defects is longer than that for divacancies, the electron density in the defects is lower than that in divacancies. The decrease in $S$ due to HAT, therefore, cannot be attributed to the decrease in the open volume of the defects due to $\mathrm{H}$ decoration. To more fully understand the annihilation characteristic of positrons trapped by H-terminated defects, it is necessary to perform further experiments and theoretical calculations on the momentum distribution of electrons in such defects.

Asoka-Kumar et al. ${ }^{14}$ and Schut et al. ${ }^{17}$ reported that effect of HAT on the $S$ value for Si without ion implantation, and they found an increase in the value of $S$ near the surface. This was attributed to the trapping of positrons by voids or nanocavities introduced by HAT. In the present experiments, since hydrogen atoms were introduced into the $\mathrm{Si}^{+}$-implanted sample, the interaction between hydrogen and pre-existing multivacancies suppresses the formation of voids or nanocavities. ${ }^{29}$ Platelets (or amorphous phase) are likely to be introduced into the region containing a very high concentration of hydrogen. As shown in Fig. 2, however, the second block (depth: $10-100 \mathrm{~nm}$ ) is deeper than the depth at which the hydrogen concentration peaks appear. In the present experiments, the effect of the trapping of positrons by platelets on the $S$ value cannot be completely neglected, but major defects produce a small $S$ value (second block) are considered to be $\mathrm{H}$-terminated vacancies.

\section{B. Annealing behavior of hydrogen-terminated vacancies}

The as-implanted and HAT samples were isochronally annealed in the temperature range between 190 and $800{ }^{\circ} \mathrm{C}$. Figure 4 shows the $S-E$ curves for the samples annealed at $385-700{ }^{\circ} \mathrm{C}$. For the sample without HAT, after annealing at $385{ }^{\circ} \mathrm{C}$, the $S-E$ curve observed is similar to that for the as-implanted sample (Fig. 1). In the case of annealing at 490 ${ }^{\circ} \mathrm{C}$, the value of $S$ at $E \cong 2 \mathrm{keV}$ decreased, suggesting a decrease in the concentration or the size of the open volume of vacancy-type defects in the subsurface region. The damaged region shifts towards the surface after annealing at $600{ }^{\circ} \mathrm{C}$, and because of the clustering of defects, the value of $S$ in the subsurface region increased. After annealing at $700{ }^{\circ} \mathrm{C}$, a decrease in the $S$ value at $E=2-3 \mathrm{keV}$ was observed, suggesting that almost all defects introduced by implantation were annealed out. With regard to the HAT sample, after annealing at $385{ }^{\circ} \mathrm{C}$, the influence of the $\mathrm{H}$ termination of vacancies on $S$ is clearly seen. After being annealed at 490 ${ }^{\circ} \mathrm{C}$, the defects located in the deeper region (defects without $\mathrm{H}$ termination) were annealed out. In the case of annealing at

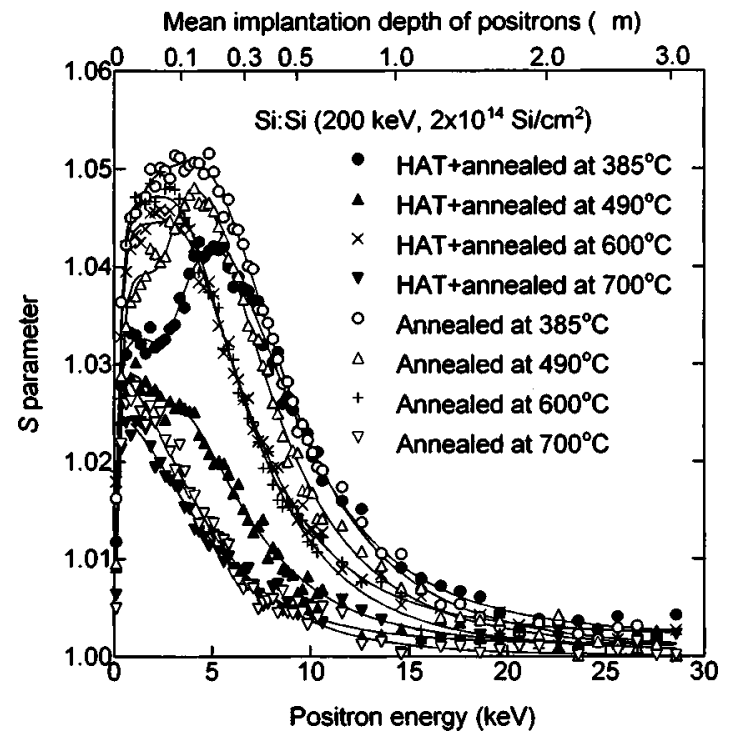

FIG. 4. $S-E$ curves for $\mathrm{Si}^{+}$-implanted $\mathrm{Si}$ annealed at $385-700{ }^{\circ} \mathrm{C}$. For the HAT sample, the difference in the values of $S$ observed after annealing at 490 and $600{ }^{\circ} \mathrm{C}$ was attributed to the release of hydrogen from vacancy-type defects at $600{ }^{\circ} \mathrm{C}$ annealing. The solid curves are fits of Eq. (1) to the experimental data.

600 and $700{ }^{\circ} \mathrm{C}$, the $S-E$ curves for the HAT sample are almost identical to those for the non-HAT sample. Figure 5 summarizes the annealing behavior of the $S$ value at $E$ $=2 \mathrm{keV}$ for the samples before and after HAT.

Figure 6 shows Raman spectra for the HAT sample after annealing at various temperatures. The vibrational line at $3820 \mathrm{~cm}^{-1}$, which is attributed to $\mathrm{H}_{2}$ molecules trapped by H-terminated vacancies or those adjacent to such defects, ${ }^{8}$ disappeared after annealing at $280^{\circ} \mathrm{C}$. The positron annihilation parameters, however, did not significantly change after annealing at this temperature. Thus, positrons are unlikely to be trapped by defects containing $\mathrm{H}_{2}$ molecules. After annealing above $600{ }^{\circ} \mathrm{C}$, all the Raman peaks arising from $\mathrm{H}_{2}$ and

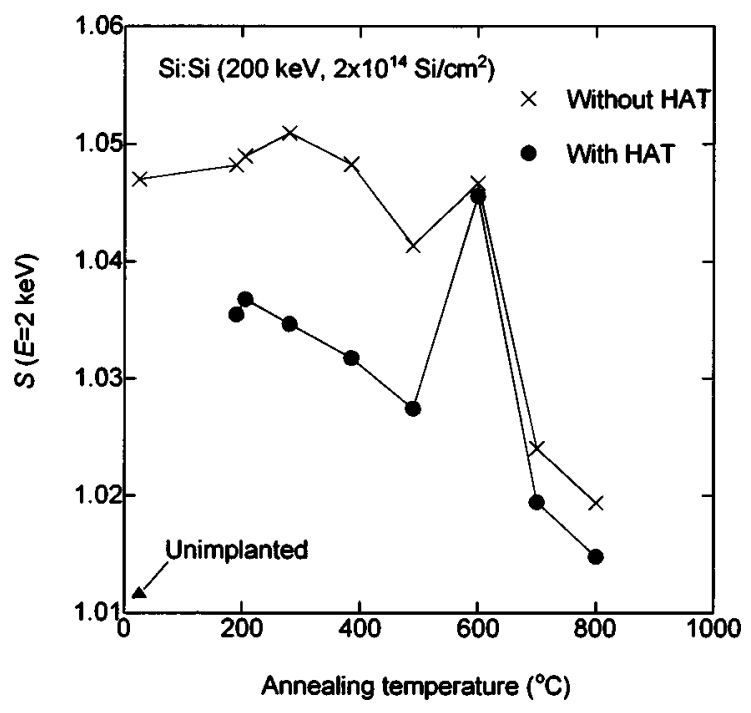

FIG. 5. Annealing behavior of the value of $S$ at $E=2 \mathrm{keV}$ for $\mathrm{Si}^{+}$-implanted $\mathrm{Si}$ with and without HAT. 


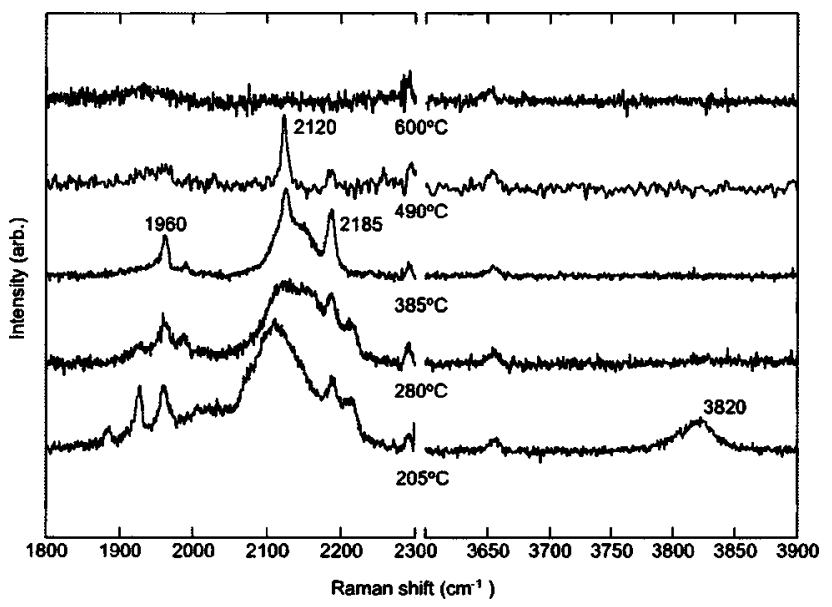

FIG. 6. Raman spectra for $\mathrm{Si}^{+}$-implanted $\mathrm{Si}$ with HAT. The vibrational line at $3820 \mathrm{~cm}^{-1}$ is due to $\mathrm{H}_{2}$ molecules trapped by $\mathrm{H}$-terminated vacancies or those adjacent to such defects (see Ref. 8). Several Raman lines observed at $1850-2250 \mathrm{~cm}^{-1}$ are associated with $\mathrm{Si}-\mathrm{H}$ bonds.

$\mathrm{Si}-\mathrm{H}$ bonds disappeared. Thus, the increase in the value of $S$ after annealing at $600{ }^{\circ} \mathrm{C}$ can be attributed to the release of hydrogen from vacancy-type defects.

For the $S-E$ curves obtained, analysis similar to that described in Sec. III A was performed. For the sample before HAT, the location between the damaged region and the defect-free region, $D_{1 / 2}$, was $360 \mathrm{~nm}$, and it starts to shift towards the surface with annealing at $490{ }^{\circ} \mathrm{C}$ (the value of $D_{1 / 2}$ for the sample annealed at $600{ }^{\circ} \mathrm{C}$ was $150 \mathrm{~nm}$ ). For the HAT sample, after being annealed at $490{ }^{\circ} \mathrm{C}$, the region of "pure" vacancies disappeared, and the region of $\mathrm{H}$-terminated defects was $0-160 \mathrm{~nm}$. In the case of annealing at $600{ }^{\circ} \mathrm{C}$, the position of the interface between the damaged and defect-free regions agrees with that in the non-HAT sample.

Using similar samples used in the present experiments, the annealing behavior of Raman spectra for $\mathrm{Si}^{+}$implanted Si after HAT was reported by Mori et al. ${ }^{29}$ In their experiments, after annealing at $610^{\circ} \mathrm{C}$ (for $30 \mathrm{~min}$ ), the sample was hydrogenated again. After this rehydrogeneration, a vibrational line was observed at around $3820 \mathrm{~cm}^{-1}$. The present experiments suggest that, after the release of hydrogen, vacancy-type defects still exist even after $600{ }^{\circ} \mathrm{C}$ annealing. Thus, the line at $3820 \mathrm{~cm}^{-1}$ can be attributed to $\mathrm{H}_{2}$ trapped by residual defects. In Fig. 6, the lines observed at 1960 and $2185 \mathrm{~cm}^{-1}$ disappeared after annealing at $490{ }^{\circ} \mathrm{C}$. At this annealing temperature, the pure vacancies located below the region of H-terminated vacancies were annealed out. The migration of $\mathrm{Si}$ atoms caused by the annealing out of such defects could change the configuration of atoms near $\mathrm{Si}-\mathrm{H}$ bonds corresponding to 1960 and $2185 \mathrm{~cm}^{-1}$.

\section{SUMMARY}

Hydrogen-terminated vacancies in $\mathrm{Si}^{+}$-implanted $\mathrm{FZ} \mathrm{Si}$ were investigated. After ion implantation into Si samples, hydrogen atoms were introduced as neutral atoms by HAT. A monoenergetic positron beam was used to obtain Doppler broadening spectra of the annihilation radiation as a function of the incident positron energy. A pulsed monoenergetic positron beam was used to obtain the lifetime spectra of positrons. The value of the line shape parameter, $S$, was increased by ion implantation, suggesting the annihilation of positrons trapped by vacancy-type defects; the mean size of the open volume of such defects was close to that of divacancies. It was found that HAT decreases the value of $S$ corresponding to the annihilation of positrons in the subsurface region (depth: 10-100 nm). The depth profile of hydrogen atoms measured by SIMS agrees well with the region in which the value of $S$ is small. Measurements of the Raman spectra show several vibrational lines that correspond to $\mathrm{Si}-\mathrm{H}$ bonds. Thus, the decrease in $S$ is attributed to the annihilation of positrons trapped by $\mathrm{H}$-terminated vacancies. The origin of the decrease in $S$ is the increase in the annihilation probability of positrons with localized electrons in $\mathrm{H}$-decorated multivacancies.

Isochronal annealing experiments were performed in the temperature range of $205-800{ }^{\circ} \mathrm{C}$. It was found that $S$ did not significantly change after annealing below $385{ }^{\circ} \mathrm{C}$, but after annealing at $490{ }^{\circ} \mathrm{C}$, almost all vacancy-type defects located below the $\mathrm{H}$-rich region were annealed out. Annealing at $600{ }^{\circ} \mathrm{C}$ results in similar $S-E$ curves for both the samples with and without HAT. These results were in good agreement with the thermal behavior of the Raman spectrum which suggests the release of hydrogen from vacancy-type defects at $600{ }^{\circ} \mathrm{C}$. The present results show that a monoenergetic positron beam can be used to study the behavior of hydrogen in $\mathrm{Si}$. The potential of positron annihilation for detecting $\mathrm{H}$-terminated vacancies located in the subsurface region and that of Raman spectroscopy for identifying various $\mathrm{Si}-\mathrm{H}$ stretching modes makes these techniques a powerful tool for studying the correlation between hydrogen and process-induced defects.

${ }^{1}$ Hydrogen in Semiconductors, edited by J. I. Pankove and N. M. Johnson (Academic, New York, 1991).

${ }^{2}$ A. J. Auberton-Hervé, M. Bruel, B. Aspar, C. Maleville, and H. Moriceau, IEICE Trans. Electron. E80-C, 358 (1997).

${ }^{3}$ K. Sakaguchi, N. Sato, K. Yamagata, T. Atoji, Y. Fujiyama, J. Nakayama, and T. Yonehara, IEICE Trans. Electron. E80-C, 378 (1997).

${ }^{4}$ T. Hashizume, S. Heike, M. Lutwyche, S. Watanabe, K. Nakajima, T. Nishi, and Y. Wada, Jpn. J. Appl. Phys., Part 2 35, L1085 (1996).

${ }^{5}$ C. G. Van de Walle, Phys. Rev. Lett. 80, 2177 (1998).

${ }^{6}$ B. Hourahine, R. Jones, S. Öberg, R. C. Newman, P. R. Beriddon, and E. Roduner, Phys. Rev. B 57, R12666 (1998).

${ }^{7}$ K. Ishioka, M. Kitajima, S. Tateishi, K. Nakanoya, N. Fukata, T. Mori, K. Murakami, and S. Hishita, Phys. Rev. B 60, 10852 (1999).

${ }^{8}$ K. Murakami, K. Ishioka, M. Kitajima, S. Tateishi, K. Nakanoya, T. Mori, and S. Hishita, Physica B 273-274, 188 (1999).

${ }^{9}$ T. Akiyama and A. Oshiyama, J. Phys. Soc. Jpn. 70, 1627 (2001).

${ }^{10}$ C. G. Van de Walle and J. Neugebauer, Phys. Rev. B 52, R14320 (1995).

${ }^{11}$ R. Krause-Rehberg and H. S. Leipner, Positron Annihilation in Semiconductors, Solid-State Sciences Ser. 127 (Springer, Berlin, 1999).

${ }^{12}$ J. Keinonen, et al. Phys. Rev. B 37, 8269 (1988).

${ }^{13}$ R. S. Brusa, M. Duarte Naia, A. Zecca, C. Nobili, G. Ottaviani, R. Tonini, and A. Dupasquier, Phys. Rev. B 49, 7271 (1994).

${ }^{14}$ P. Asoka-Kumar, H.J. Stein, and K.G. Lynn, Appl. Phys. Lett. 64, 1684 (1994)

${ }^{15}$ M. Fujinami, R. Suzuki, T. Ohdaira, and T. Mikado, Phys. Rev. B 58, 12559 (1998). 
${ }^{16}$ C. E. Gonzalez, S. C. Sharma, N. Hozhabri, D. Z. Chi, and S. Ashok, Appl. Phys. A: Mater. Sci. Process. 68, 643 (1999).

${ }^{17}$ H. Schut, A. van Veen, S. W. H. Eijt, R. Job, A. G. Ulyasin, and W. R. Fahrner, Nucl. Instrum. Methods Phys. Res. B 186, 94 (2002).

${ }^{18}$ A. van Veen, H. Schut, J. de Vries, R. A. Hakvoort, and M. R. Ijpma, AIP Conf. Proc. 218, 171 (1990).

${ }^{19}$ A. Uedono, T. Kitano, K. Hamada, T. Moriya, T. Kawano, S. Tanigawa, R. Suzuki, T. Ohdaira, and T. Mikado, Jpn. J. Appl. Phys., Part 1 36, 2571 (1997).

${ }^{20}$ R. Suzuki, T. Ohdaira, and T. Mikado, Radiat. Phys. Chem. 58, 603 (2000).

${ }^{21}$ P. Kirkegaard, M. Eldrup, O. E. Mogensen, and N. J. Pedersen, Comput. Phys. Commun. 23, 307 (1981).

${ }^{22}$ M. J. Puska and C. Corbel, Phys. Rev. B 38, 9874 (1988).
${ }^{23}$ M. Saito and A. Oshiyama, Phys. Rev. B 53, 7810 (1996).

${ }^{24}$ J. W. Corbett, J. P. Karins and T. Y. Tan, Nucl. Instrum. Methods Phys. Res. 182/183, 457 (1981).

${ }^{25}$ A. Uedono, Y. Ujihira, A. Ikari, H. Haga, and O. Yoda, Hyperfine Interact. 79, 615 (1993).

${ }^{26}$ A. Uedono, M. Watanabe, S. Takasu, T. Sabato, and S. Tanigawa, J. Phys.: Condens. Matter 12, 719 (2000).

${ }^{27}$ Z. Tang, T. Nonaka, Y. Nagai, and M. Hasegawa, Mater. Sci. Forum 363365, 67 (2001).

${ }^{28}$ M. Hasegawa, Z. Tang, Y. Nagai, T. Nonaka, and K. Nakamura, Surf. Sci. 194, 76 (2002).

${ }^{29}$ T. Mori, K. Otsuka, N. Umehara, K. Ishioka, M. Kitajima, S. Hishita, and K. Murakami, Physica B 302-303, 239 (2001). 